\title{
Diacronie
}

Studi di Storia Contemporanea

$N^{\circ} 28,4 \mid 2016$

La voce del silenzio

\section{Marten Düring, Verdeckte soziale Netzwerke im Nationalsozialismus. Die Entstehung und Arbeitsweise von Berliner Hilfsnetzwerken für verfolgte Juden}

Reti sociali clandestine nel nazionalsocialismo. La costituzione ed il funzionamento delle reti di aiuto per perseguitati ebrei a Berlino

\section{Daniele Toro}

\section{OpenEdition}

\section{Journals}

Edizione digitale

URL: http://journals.openedition.org/diacronie/4524

DOI: $10.4000 /$ diacronie. 4524

ISSN: 2038-0925

Editore

Association culturelle Diacronie

Notizia bibliografica digitale

Daniele Toro, «Marten Düring, Verdeckte soziale Netzwerke im Nationalsozialismus. Die Entstehung und Arbeitsweise von Berliner Hilfsnetzwerken für verfolgte Juden », Diacronie [Online], № 28, 4 | 2016, Messo online il 29 décembre 2016, consultato il 24 septembre 2020. URL : http://journals.openedition.org/ diacronie/4524; DOI : https://doi.org/10.4000/diacronie.4524 


\section{Diacronie}

N. 28 | 4|2016 La voce del silenzio: intelligence, spionaggio e conflitto nel XX secolo

\section{0/}

\section{PANORAMICA: Germania 2015}

Silvia MADOTTO, Alessandro SALVADOR, Daniele TORO *

WOLLER, Hans, Mussolini. Der erste Faschist. Eine Biographie [Mussolini. Il primo fascista. Una biografia], München, C.H. Beck Verlag, 2015, 397 pp.

a cura di Alessandro SALVADOR

La vita di Benito Mussolini, il fascismo e la Seconda guerra mondiale occupano una posizione ancora scomoda nella memoria storica italiana. È un po' questo il pensiero dominante della biografia realizzata da Hans Woller. La stessa definizione di biografia, per questo volume, è decisamente riduttiva. Attraverso undici date e luoghi chiave della storia di Mussolini e del fascismo, Woller riesce in un'impresa non semplice: sintetizzare il più recente e completo stato della ricerca su quel periodo storico, esporlo in modo chiaro e con un linguaggio accattivante e, non ultimo, esprimere in modo non invadente alcune riflessioni personali, risultato di un lungo percorso di studi e ricerche sul tema.

Il percorso di Woller si snoda attraverso momenti chiave della vita del Duce, dalla sua formazione politica alla morte a Giulino di Mezzegra, scandendone momenti importanti come la fase socialista, la creazione del fascismo, la dittatura e l'impero e la controversa e tutt'altro che semplice alleanza con Hitler. La struttura dei capitoli sembra ricalcare, con le definizioni, la ben nota biografia in più tomi di Renzo de Felice. Ma le analogie si fermano qui. Woller dipinge un Mussolini cinico e opportunista.

Nel volume sono affrontate tematiche del fascismo che spesso passano in secondo piano o sono state affrontate dalla storiografia solo in tempi recenti. 
L'immagine del Duce viene riportata nel suo contesto storico, ridimensionando i miti, in negativo o in positivo, che l'hanno caratterizzata in passato. Ed è qui che il volume di Woller offre una prospettiva interessante, nel rimettere le figura di Mussolini, il suo sviluppo e le sue imprese, in un contesto storico. Nell'evidenziare la futilità e le brutalità delle imprese coloniali, la vanità della guerra parallela e l'incessante aspirazione a distruggere l'ordine europeo scaturito dai trattati successivi alla Grande guerra.

L'ammirazione che in un primo momento Mussolini suscitò in ambienti liberali, la sua iniziale posizione anti-tedesca, la presunta umanità della condotta di guerra italiana rispetto a quella nazista vengono ricontestualizzati grazie all'uso di una vasta letteratura scientifica e a un buon uso delle fonti.

In sostanza, questo volume è un ottimo compendio delle conoscenze su Mussolini e il fascismo che può fornire spunti interessanti al lettore esperto ed una sintesi molto leggibile al semplice curioso.

SCHMIDT, Daniel, STURM, Michael, LIVI, Massimiliano (Hrsg.), Wegbereiter des Nationalsozialismus: Personen, Organisationen, Netzwerke der extremen Rechten zwischen 1918 und 1933 [I precursori del nazionalsocialismo: persone, organizzazioni e reti dell'estrema destra tra il 1918 e il 1933], Essen, Klartext, 2015,290 pp.

a cura di Alessandro SALVADOR

Il volume offre una selezione di saggi sull'estrema destra tedesca nella Repubblica di Weimar. I contributi proposti si focalizzano sulle continuità e discontinuità tra il cosiddetto milieu nazionalista radicale e il nazionalsocialismo.

Il volume è suddiviso in quattro parti, ognuna delle quali presenta diversi contributi. Nella prima sezione, dedicata alle formazioni völkisch, emergono alcuni aspetti poco noti dei legami tra alcuni movimenti e il nazionalsocialismo. Interessante la prospettiva focalizzata su un singolo personaggio politico, con l'analisi della storia personale di Max Robert Gerstenhauer.

I saggi sulle formazioni paramilitari offrono prospettive molto interessanti rispetto alla tradizione storiografica precedente. Le analisi della provenienza sociale dei volontari dei Freikorps, i legami tra le associazioni studentesche e il nazionalsocialismo, nonché le prospettive internazionali e transnazionali esposte 
impongono un ripensamento dei rapporti tra paramilitari, ceti medi e conservatori e nazionalsocialismo.

Nella terza parte vengono analizzati casi regionali con una particolare attenzione alle reti di organizzazioni e di persone, offrendo nuovi orizzonti metodologici alla ricerca delle correlazioni tra gruppi di interesse e di pressione e il complesso panorama politico repubblicano.

Infine, la prospettiva di genere presentata dagli ultimi due saggi aggiunge un ulteriore elemento di novità e di distacco dalla storiografia precedente che, su queste tematiche, era ancora legata a cornici interpretative classiche.

In sostanza, un volume ricco di spunti interessanti e nuove ricerche che non mancherà di stimolare nuovi studi sulla politica nel periodo interbellico.

DÜRING, Marten, Verdeckte soziale Netzwerke im Nationalsozialismus. Die Entstehung und Arbeitsweise von Berliner Hilfsnetzwerken für verfolgte Juden [Reti sociali clandestine nel nazionalsocialismo. La costituzione ed il funzionamento delle reti di aiuto per perseguitati ebrei a Berlino], Berlin, De Gruyter Oldenbourg, 2015, 215 pp.

a cura di Daniele TORO

L'analisi delle pratiche di sostegno in favore degli ebrei perseguitati durante il nazionalsocialismo è stata spesso caratterizzata dalla rappresentazione idealtipica del perseguitato come oggetto ricettore delle prestazioni di supporto fornitegli da una controparte pienamente attiva, non di rado a sua volta ritratta con tinte agiografiche.

La pubblicazione della tesi di dottorato di Marten Düring, incentrata sulla reinterpretazione in chiave relazionale di attori, pratiche e strutture implicate nel prestare soccorso ai perseguitati ebrei, offre un modello alternativo a tale univocità. Lo studio è condotto attraverso l'applicazione dell'analisi storica delle reti sociali al contesto dei network clandestini di supporto berlinesi, e rappresenta in tal senso il primo tentativo di farne un uso estensivo e sistematico all'interno del proprio campo di ricerca. Nell'introduzione l'autore descrive attraverso una metanarrazione il processo euristico condotto per applicare la teoria dell'analisi dei network al caso studio in analisi, fornendo in tal senso una stimolante riflessione metodologica.

Il rilevamento dei dati quantitativi necessari all'indagine è effettuato da Düring a partire da uno spettro variegato di fonti, consolidato da verifiche incrociate delle informazioni raccolte. La parte centrale della trattazione, strutturata sulla base di sei casi specifici, è dedicata alla ri- e alla decostruzione empirica delle reti di aiuto sul 
piano microanalitico, le cui linee di sviluppo comune vengono infine messe in evidenza nella terza ed ultima sezione. Qui Düring pone a confronto i risultati dei singoli casi studio delineandone le dinamiche generalizzabili e giungendo a stabilire che, nonostante il limitato spazio d'azione loro concesso dalle strutture repressive statali, $\mathrm{i}$ perseguitati disponessero di un'agency funzionale e necessaria alla costituzione ed allo sviluppo dei network di supporto.

\section{GAMPER, Marcus, RESCHKE, Linda, DÜRING, Marten (Hrsg.), Knoten} und Kanten III. Soziale Netzwerkanalyse in Geschichts- und Politikforschung [Vertici e lati III. L'analisi delle reti sociali nella ricerca storica e politica], Bielefeld, Transcript, 2015, 426 pp.

a cura di Daniele TORO

Dal principio del XXI secolo si è assistito ad un aumento esponenziale degli studi dedicati all'analisi delle reti sociali, la cui portata transdisciplinare ha coinvolto negli ultimi anni il panorama storiografico tedesco in modo particolarmente vivace.

Inserita in tale contesto, la pubblicazione del terzo titolo collettaneo appartenente alla serie "Knoten und Kanten" rappresenta una conferma della progressiva affermazione ed istituzionalizzazione dell'analisi dei network in campo storiografico. Il volume raccoglie in tre diverse sezioni testi funzionali all'illustrazione delle potenzialità euristiche dell'analisi delle reti sociali applicata alle scienze storiche e politiche. In tal senso all'introduzione dei curatori segue un primo blocco di saggi a carattere teorico, dedicati alla definizione dell'analisi delle reti sociali sulla base di problematiche specifiche quali, ad esempio, il rapporto con l'ascendente paradigma relazionale o la sua possibile integrazione con un'indagine storico-sociale dei rapporti di potere. Alle possibili declinazioni empiriche di tali teorie sono invece dedicati - rispettivamente nella seconda e terza sezione - i casi studio delineati nei saggi a carattere storico e politico. I contributi storici, introdotti da una riflessione sulla dimensione temporale dei network, si dividono tra una panoramica delle sue applicazioni alla storia antica e tre studi in storia economica e sociale dell'età contemporanea.

Il volume si presta pertanto, anche in virtù della propria natura collettanea, ad essere recepito, su un doppio livello di lettura, tanto come introduzione scientifica

\footnotetext{
${ }^{1}$ Per i titoli precedenti cfr. GAMPER, Marcus, RESCHKE, Linda (a cura di), Knoten und Kanten. Soziale Netzwerkanalyse in Wirtschafts- und Migrationsforschung, Bielefeld, Transcript, 2010; GAMPER, Marcus, RESCHKE, Linda, SCHÖNHUT, Michael (a cura di), Knoten und Kanten 2.o. Soziale Netzwerkanalyse in Medienforschung und Kulturanthropologie, Bielefeld, Transcript, 2012.
} 
quanto come raccolta di approfondimenti funzionali ad una riflessione più mirata ed innovativa riguardo a temi e problematicità irrisolti all'interno un campo di ricerca ancora in divenire.

SCHMALE, Wolfgang (Hrsg.), Digital Humanities. Praktiken der Digitalisierung, der Dissemination und der Selbstreflexivität [Digital Humanities. Pratiche di digitalizzazione, disseminazione $e$ autoriflessione], Stuttgart, Steiner, 2015, $183 \mathrm{pp}$.

a cura di Silvia MADOTTO

Wolfgang Schmale, professore di Storia contemporanea presso l'Università di Vienna, raccoglie, in questo volume collettaneo, contributi che affrontano complesse e dibattute tematiche nell'ampio ambito delle Digital Humanities. Ciò che connette e accomuna i distinti saggi è soprattutto una riflessione sul potenziale di novità che le DH possono portare concretamente nella ricerca, dunque i tangibili cambiamenti nella teoria e nella prassi delle scienze umane. Nella prima sezione del libro sono discusse, tra vantaggi e problematiche, le metodologie e le tecniche digitali di raccolta e utilizzo di dati e fonti digitali/digitalizzate, tra cui le fonti audio. Nella sezione centrale i contributi si concentrano sull'analisi della divulgazione dei dati scientifici attraverso il digitale e sulla discussione dei cambiamenti che questi nuovi formati digitali portano nella comunicazione scientifica. Al centro della discussione sono posti l'utilizzo dei big data nella ricerca, ma anche Wikipedia, i weblogs, le Smartphone-apps fino alla realtà virtuale dei giochi per computer. Riflessioni sull'utilizzo delle edizioni digitali, sul linguaggio e l'interpretazione del mondo digitale, la codinghistory e linguaggi di programmazione, chiudono il volume. Nei diversi contributi trovano spazio temi complessi e controversi che animano da anni il dibattito sulle DH: obiettivo del volume è stimolare tali dibattiti e riflessioni sul digitale nella ricerca scientifica umanistica. Come rimarca il curatore, il lavoro nelle DH ha davanti a sé ancora un lungo percorso.

STEINBACH, Peter, Nach Auschwitz. Die Konfrontation der Deutschen mit der Judenvernichtung [Dopo Auschwitz. Il confronto dei tedeschi con lo sterminio degli ebrei], Bonn, Dietz, 2015, 107 pp.

a cura di Silvia MADOTTO

Peter Steinbach, professore emerito e direttore del Centro di ricerca e commemorazione della resistenza tedesca di Berlino, elabora questo saggio muovendo 
da riflessioni sulla Giornata della Memoria per le vittime del nazionalsocialismo e lo sterminio degli ebrei. Il testo, dal carattere di riflessione soggettiva, è permeato da spunti di riflessione sul ruolo della commemorazione e della cultura storica per la formazione politica e civile contemporanea. L'autore, focalizzandosi sull'importanza della lotta per la verità storica, ripercorre le diverse fasi da Weimar al processo Eichmann in Israele. Arrivando alla Germania contemporanea, Steinbach si muove tra il mutare dei ricordi e la memoria del passato nazista, fino alle riflessioni sulla retorica e l'uso del linguaggio in discorsi politici contemporanei. Toccando con richiami la contemporaneità mondiale tra Africa, Medio Oriente, Balcani e concentrandosi sulla Germania di oggi, Steinbach riflette su come la cultura storica possa offrire oggi importanti basi di valori e riferimenti, discutendo poi la possibilità di rispettare la promessa del "mai più". In un panorama mondiale costellato da continui casi di emarginazione ed esclusione e in cui il genocidio non è solo una realtà storica, ma tocca il presente e si compie davanti ai nostri occhi, oggi l'importanza del ricordare è più che mai viva. Obiettivo della Giornate della Memoria è dunque di avvicinare, coinvolgere, ma anche invitare a riflettere i cittadini sulle sfide della civiltà moderna e gli sviluppi politici. Contro il decorso della memoria Steinbach ribadisce quanto sia fondamentale la commemorazione, la discussione, la fatica del ricordare. 


\section{* Gli autori}

Silvia Madotto è dottoranda alla Freie Universität di Berlino. Ha studiato storia antica e contemporanea presso le Università di Udine, Padova e Berlino. La sua tesi indaga forme e organizzazioni di antifascismo e Resistenza presso le Università in Europa durante la Seconda guerra mondiale. I suoi interessi includono: storia transnazionale, fascismo e antifascismo in Europa, storia delle Università, storia italiana del Ventesimo secolo. Ha pubblicato un numero monografico della rivista «Storia contemporanea in Friuli», dal titolo Le capitali della Resistenza universitaria. Padova, Oslo e Praga ed è in corso di pubblicazione un articolo su Silvio Trentin e l'antifascismo in Europa (atti del convegno "Fascism without Borders. Transnational Connections and Cooperation between Movements and Regimes in Europe from 1918 to $1945 ”)$.

URL: < http://www.studistorici.com/progett/autori/\# Madotto >

Alessandro Salvador si è laureato in Storia Contemporanea all'Università di Trieste nel 2006 e, nel 2010, ha conseguito il Dottorato di Ricerca in Studi Storici presso l'Università di Trento con una tesi dal titolo "Il partito nazionalosocialista e la destra radicale tedesca nelle fasi finali della Repubblica di Weimar: 1925-1933". Tra le sue pubblicazioni vi sono il volume La guerra in tempo di pace. Gli ex combattenti e la politica nella Repubblica di Weimar (Trento, Università degli Studi di Trento, 2013) e alcuni saggi sul combattentismo e sulla smobilitazione dei soldati ex austro-ungarici in Italia dopo la Grande guerra. Attualmente è assegnista di ricerca presso l'Università di Trento e collabora col progetto www.lagrandeguerrapiu10o.it, col progetto europeo CENDARI (Collaborative European Digital ArchivalInfrastructure) e col progetto “World War II - Everyday Life Under GermanOccupation.” dell'HerderInstitut di Marburg.

URL: < http://www.studistorici.com/2015/10/16/alessandro_salvador/ >

Daniele Toro ha conseguito la Laurea triennale in Storia presso l'Università di Bologna. Iscritto al corso di Laurea magistrale in Scienze storiche presso lo stesso ateneo, studia dal 2013 presso l'Universität Bielefeld all'interno del Corso di Laurea magistrale integrato italo-tedesco in Scienze storiche (BiBoG). I suoi interessi di ricerca vertono sulla teoria della storia e l'analisi comparata dei fascismi.

URL: < http://www.studistorici.com/progett/autori/\#Toro > 


\section{Per citare questo articolo:}

MADOTTO, Silvia, SALVADOR, Alessandro, TORO, Daniele, «Panoramica: Germania 2015», Diacronie. Studi di Storia Contemporanea : La voce del silenzio: intelligence, spionaggio e conflitto nel XX secolo, 29/12/2016, URL:<http://www.studistorici.com/2016/12/29/germania_numero_28/ >

Diacronie Studi di Storia Contemporanea $\vartheta$ www.diacronie.it

Risorsa digitale indipendente a carattere storiografico. Uscita trimestrale. redazione.diacronie@hotmail.it

Comitato di redazione: Jacopo Bassi - Luca Bufarale - Antonio César Moreno Cantano - Deborah Paci - Fausto Pietrancosta - Alessandro Salvador - Matteo Tomasoni - Luca Zuccolo

Diritti: gli articoli di Diacronie. Studi di Storia Contemporanea sono pubblicati sotto licenza Creative Commons 3.0. Possono essere riprodotti e modificati a patto di indicare eventuali modifiche dei contenuti, di riconoscere la paternità dell'opera e di condividerla allo stesso modo. La citazione di estratti è comunque sempre autorizzata, nei limiti previsti dalla legge. 